\title{
L'aliené devant la philosophie, la morale et la société*1
}

\section{The mentally ill seen from perspective of philosophy, ethics and society}

Albert Lemoine*2

\author{
Chapitre Premier
}

\section{Préjuges et questions sur la folie}

Il existe aujourd'hui dans presque tous nos départements des maisons spéciales, où les malheureux qui ont perdu la raison trouvent un asile et de soins, où ils vont soit recouvrer leur esprit égaré, soit achever le plus doucement possible leur vie misérable. Là sont réunis dans une même enceinte des furieux qui crient et gesticulent, des malades paisibles qui promènent éternellement leur mélancolie, ou font retentir les salles et les préaux des éclats d'une gaieté navrante. Là encore végètent de pauvres êtres dont l'intelligence parait éteinte, vivant comme des bêtes, sans que leur esprit soit ou semble occupé d'une seule pensé, jeunes ou vieux, enfants de tous les âges. Sur celles de

*1 Librairie Academique. Paris: Didier et Cie. Libraires-Éditeurs, 1862.

*2 Professeur de Philosophie au Licée Bonaparte. 


\section{CLÁSSICOS DA PSICOPATOLOGIA}

ces maisons qu'ouvre la charité publique, on lit cette inscription: Asile des aliénées, on y lisait naguère: Hospice des fous.

Avant de demander aux savants quelle est la nature du mal dont sont affligés les tristes hôtes de ces asiles, quelle est leur état physique et intellectuel, quelle est la différence qui les sépare de nous, quelle est leur condition dans la société et devant la morale, demandons nous a nous-mêmes quelles idées nous nous faisons de toute ces choses, et nous nous apercevrons, après quelques moments de réflexion, que notre esprit n'est plein que de notions vagues et confuses, ou de préjugés ignorants et contradictoires. Sera-ce une consolation de découvrir que le commun des hommes, que les esprits les plus distingués eux-mêmes, je mets à part les médecins spéciaux mais non les philosophes, ne sont pas plus savants que nous, et ne conçoivent pas de la folie des idées plus claire et plus précises?

Rien n'est plus aisé que de nous en convaincre. Il suffit de rechercher quelle et la signification attachée par l'usage a une dizaine de termes employés par le plus grand nombre, ou par le nombre plus restreint des maitres de la langue, pour savoir non pas quelle est la vérité sur la folie, quelle est réellement la nature de ce mal, mais quelles idées s'en forment le vulgaire et les esprits d'élite.

Les mots folie, démence, délire, manie, aliénation mentale, fou, insensé, aliéné, sont les termes les plus fréquemment usités pour désigner l'état des habitants des asiles, ou ces malheureux eux-mêmes. Le moyen le plus simple, et qui s'offre tout d'abord pour définir le sens d'un mot de notre langue, est de consulter le dictionnaire de l'Académie française. Je l'ouvre et je lis: «Folie, démence, aliénation d'esprit. — Démence, folie, aliénation d'esprit. Aliénation d'esprit ou aliénation mentale, égarement d'esprit, folie. — Delire, égarement d'esprit causé par la maladie. - Manie, folie qui n'est pas complète comme la démence, et qui se manifeste par des accès intermittents. - Fou, qui a perdu le sens, l'esprit - Insensé, fou, qui a perdu le sens, qui a l'esprit aliéné. - Aliéné, s'emploie substantivement et absolument pour désigner ceux qui sont fous, qui ont perdu l'esprit —». Faut-il prendre ces définitions à la rigueur, en presser la lettre et comprendre que, tandis que le délire aurait sa cause dans un mal corporel, la folie, l'aliénation mentale ou la démence serait pour le plus grand nombre un égarement de l'esprit tout à fait indépendant de l'état des organes? Ne faut-il pas penser bien plutôt que la multitude ne croit pas en tant dire avec si peau de mots, qu'elle n'exprime ni ne professe une théorie si précise, quelle qu'en soit l'erreur ou la vérité, quand elle fait usage de ces termes, et que les définitions en sont indécises et banales? 
Cherchons ailleurs, et puisque plusieurs de ces mots passent pour synonymes, consultons un dictionnaire de synonyme celui, par exemple, de M. Lafaye. Il ne calque point ses définitions sur l'usage commun, bourgeois, seulement coutumier, souvent inintelligent des mots de notre langue; il consulte le délicats et les puristes, n'admet pour autorités que les écrivains d'élite, et puise aux meilleures sources. Nous y devons trouver clairement énoncée l'idée que se sont faite de la folie les esprits les plus éclairés parmi nos grands écrivains, la différence reconnue et observée par la raison et le goût entre tous le termes synonymes, ou bien nous devrons penser que nos meilleurs écrivains ne se sont pas fait une idée plus précise de la folie que le vulgaire.

«Délire, égarement, folie, démence et manie, lit-on dans le dictionnaire de M. Lafaye, annoncent qu'on est hors de sens, qu'on a le cerceau malade.» Voilà, certes, une définition très claire et très-précise, bien qu'elle s'applique à la fois à un groupe de cinq mots d'étymologies différentes; elle ne s'arrête pas seulement au signe, elle atteint, elle exprime la chose signifiée elle-même; c'est presque une définition de chose, comme disent les logiciens, et non pas une simple définition de mot. En effet, plus d'un médecin se demande encore aujourd'hui si la folie est une maladie de l'esprit ou une maladie corporelle, et cette définition tranche la question en faveur d'une opinion précisément contraire à celle que semblait donner tout à I'heure l'Académie française. Elle va même plus loin dans cette voie que beaucoup de savants, et déclare que par le mot folie on entend et il faut entendre, non-seulement une maladie corporelle, quelque partie du corps, quelque organe qu'elle affecte, mais une maladie du cerveau, ce qui est encore plus douteux pour un bon nombre de physiologistes. Ainsi un simple article de dictionnaire, une définition de mot, en apparence insignifiante, nous en aurait appris plus long sur la nature de la folie, ou du moins sur l'idée que s'en font le commun des hommes et les esprits d'élite, que la lecture de bien des livres. Mais ne nous hâtons pas de conclure avant d'avoir terminé l'article. Je lis plus loin: «Folie, démence et manie expriment de maladies de l'esprit ou l'aliénation mentale.» Nous croyions tenir une idée claire, une bonne définition, et nous voilà rejetés de vive force dans le doute et l'obscurité, non-seulement sur la nature de la folie qu'un dictionnaire français n'est pas chargé de nous faire connaitre, mais sur l'idée que le mot folie représente. Ce même mot, en effet, par lequel on désignait tout à l'heure une maladie du cerveau, désigne à présent une maladie de l'esprit.

Faut-il conclure de ce double insuccès que le dictionnaire de l'Academie française est inutile, que celui de $\mathrm{M}$. Lafaye est mal fait et renferme 


\section{CLÁSSICOS DA PSICOPATOLOGIA}

des contradictions? $\mathrm{C}^{\prime}$ est une sotte mode que de mépriser le dictionnaire de l'Académie, et bonne pour ceux qui se dispensent ainsi de parler ou d'écrire la langue française; le livre de M. Lafaye fait certainement avec tout le soin et toute la science possibles et pour peu qu'on se soucie de sa langue, on doit l'avoir non pas dans sa bibliothèque, mais sous sa main. Il faut tirer de cette expérience une conclusion toute différente. Si l'Académie et M. Lafaye ne définissent pas avec plus de rigueur les mots dont nous parlons, s'ils n'expriment pas plus clairement les idées que mots désignent dans notre esprit, c'est que les idées que nous concevons généralement des choses représentées par ces termes sont elles-mêmes obscures et confuses. Si M. Lafaye, par exemple, nous dit d'abord qu'on entend par le mot folie une maladie du cerveau, puis, quelque lignes plus bas, que ce mot signifie une maladie de l'esprit, c'est que, ni la plupart de ceux qui emploient ce mot sans s'en rendre compte, ni le petit nombre de ceux qui passent pour se rendre raison de leurs idées et de leurs termes, ne savent point si la folie réellement une maladie de l'esprit ou du cerveau, que peut-être ils n'ont songé seulement qu'il y eût sous ce mot une grave question, et qu'ils n'ont pas surtout prétendu la résoudre. Loin de conclure que les dictionnaires sont mauvais parce qu'ils ne définissent pas rigoureusement ces idées et ces mots, je conclurais plutôt par cela même en leur faveur. On ne peut ni ne doit définir rigoureusement le sens d'un mot, lorsque l'idée qu'il représente est obscure, indistincte, et flotte indécise entre les deux contraires. Définir un tel mot avec rigueur, lui attribuer un sens précis et enfermé dans d'étroites limites, ce serait mettre à la place de l'idée de tous celle d'un seul, créer un mot nouveau, un terme savant, au lieu d'expliquer un mot de la langue usuelle. Avec de telles prétentions, un vocabulaire ne serait plus le trésor de tous les signes d'une nation et l'image fidèle de ses idées, un interprète juré entre tous ceux qui parlent un même idiome, que l'on consulte pour s'entendre, et qui fait cesser les équivoques; ce serait une compilation arbitraire, incompréhensible et contradictoire de signes connus et communs et d'idées individuelles, de vérités, d'erreurs et d'hypothèses, sans autorité, sans utilités, toujours contestable, propre à jeter la discorde dans les esprits sous prétexte de les instruire, capable d'accomplir dans un même langue la confusion de Babel.

La plupart n'ont de la folie que des idées vagues, confuses et souvent contradictoires. C'est un fait dont la raison est en partie dans l'histoire. L'étude et le traitement de la folie datant d'hier; d'hier seulement quelques médecins spéciaux, en petit nombre encore, ont cherché à éclairer cette partie de la science demeurée intacte et comme inviolable. Ils commencent à peine 
eux-mêmes à y voir luire quelque claré, à se faire des phénomènes qu'ils observent une idée nette, à édifier à tâtons une explication vraisemblable. Comment nous autres, le vulgaire, pourrions - nous avoir des ces choses des notions exactes, nous servir de mots rigoureusement définir pour exprimés nos idées confuses, avoir résolu les questions dont le plus savant sait à peine encore poser les termes? Du moins n'est-il pas inutile de nous bien convaincre tout d'abord de notre ignorance, de dissiper tous les préjuges qui entretiennent la confusion dans nos esprits, d'en chasser toutes les superstitions qui composent l'histoire des idées populaires sur la folie jusqu'a la fin du dernier siècle, de faire table rase de toutes les erreurs dangereuses qui ont tant nui à une multitude d'innocents malheureux, et circulent encore aujourd'hui avec les mêmes dangers.

Maintenant que le moyen âge, qui a duré si longtemps pour la médecine et surtout pour l'étude de la folie, est bien décidément fini en toutes choses, essayons de concevoir de la folie des idées plus claires, plus réfléchies, plus raisonnables; voyons quels problèmes ce mal singulier pose au médecin, au philosophe, au moraliste, au légiste. Si la folie était un mal comme un autre, sans autres conséquences que la douleur et la mort, il faudrait évidemment laisser faire aux habiles, et ne point nous mêler où nous n'aurions rien à voir. Mais il en est autrement de la folie, quelles que soient sa nature et son origine, que d'une fièvre typhoïde, sur laquelle il n'est pas urgent que nous ayons une opinion raisonnable. Dans la folie, il ne s'agite pas seulement de médecine, de traitement et de guérison, de maladie passagère ou incurable, des choses enfin qui, tout en touchant aux intérêts de tous, peuvent et doivent demeurer le fait, la province de quelques-uns. Du moment que le bon sens, la raison, la volonté, la liberté, la responsabilité de l'agent, la justice humaine sont en jeu, c'est ne plus là une question qui doive être vidée entre médecins et physiologistes; l'horizon s'élargit, la psychologie, la morale, la philosophie, en un mot, est intéressée directement et a voix au chapitre. Or le questions philosophiques et morales sont des questions populaires et du domaine public; la philosophie n'est pas une profession exercée par un petit nombre, ni mène une science accessible seulement à quelques adeptes, c'est une science permise à tous, bien mieux, obligatoire pour tout esprit cultivé. Sur les questions philosophiques et morales, tout le monde est intéressé à s'enquérir, a s'instruire, et l'opinion de quiconque a observé et médité a sa valeur; il n'y a pas de profanes. Et personne ne niera que l'étude de la folie n'appartienne aussi au philosophe, quand bien même le traitement en regarderait le seul médecin, que la folie ne soulève de graves questions de morale, qu'elle ne pose au 


\section{CLÁSSICOS DA PSICOPATOLOGIA}

légiste de difficiles problèmes, qu'elle ne touche à tous les intérêts de la famille et de la société, enfin qu'elle ne doive exciter justement la curiosité d'un esprit sérieux.

Il ne semble pas qu'il y ait des motifs suffisants pour bannir à peu près le mot de folie ou de fou, et lui préférer, dans tous les cas, celui d'aliénation ou d'aliéné qui s'est très-répandu depuis quelques années. Chacun de ces mots a son usage et ses raisons d'être. Écoutons parler deux personnes différentes des victimes du triste fléau. L'une est un homme du peuple, sans aucune prétention à la science, qui appelle les choses par leurs noms, pour qui un chou et un chou et non une crucifère ni une brassica. Il ne dit pas non plus que son voisin est atteint de démence ou d'aliénation mentale mais qu'il est fou. L'autre est un magistrat ou un médecin; pour lui un fou est un aliéné. Ils parlent cependant tous deux du même homme et de la même chose. C'est qu'il n'y a guère entre ces deux mots qu'une différence, non de sens, mais de convenance, plutôt morale et formelle que profonde et savante, qui provient moins de l'idée ou de la chose désignée que de la personne qui parle et de sa condition. Le mot aliénation est un terme médical et légal, qui représente assez bien l'état du fou vis-à-vis de lui-même, de son passé, de ses semblables; l'aliéné est autre qu'il était ou qu'il devrait être, autre que le reste des hommes, il est comme étranger à lui-même, étranger à la société politique et civile, à ses lois ordinaires et aux droits de ses membres raisonnables. Le nom d'aliéné est plus solennel, il renferme je ne sais quoi de plus délicat et comme de plus poli; l'employer de préférence à tout autre, c'est dans certaines circonstances donner au mal-heureux que l'on désigne une marque de sympathie et de respect. Qu'il soit donc le terme à peu près exclusif du magistrat et du médecin, qu'il soit seul prononcé dans le prétoire de la justice, qu'il soit inscrit comme en évidence au fronton des asiles et mêmes au titre de ce livre. Mais les mots de fou et de folie sont les mots populaires: ce que la médecin appelle des noms pompeux de bronchite et de coryza, tout le monde 1'appelle encore et tout bonnement un rhume; ce que la science nomme aliénation mentale sera toujours la folie pour tout le monde. Ce sont les idées de tout le monde que nous voudrions éclairer; pourquoi éviterions-nous de parler le langage de tous? Aussi bien s'efforcerait-on vainement de bannir ces dénominations usuelles; elles viennent se placer d'elles-mêmes sur les lèvres ou sous la plume de l'avocat ou de l'écrivain.

La folie ou l'aliénation mentale, sous ses principales formes et un mal aussi vieux que l'homme, un des plus anciennement et de plus certainement constatés par les témoignages de l'histoire, entre tous nos maux. En effet, de 
maladies qui affligent aujourd'hui l'humanité quelques-unes sont certainement nouvelles, et en échange, quelques - unes de celles qui affligeaient nos ancêtres ont disparu: la folie a persisté à travers les siècles. Peut-être parce qu'elle est réellement ou apparemment plus terrible parce que les symptômes intellectuels par lesquels elle se trahit, le délire, les visions mensongères, les dérèglements, de l'imagination, les erreurs du jugement, l'extravagance des actions ou l'insensibilité et l'abrutissement de l'intelligence, frappent d'un étonnement profond celui qui les observe, des exemples nombreux et incontestable de folie sont consignés dans les livres les plus anciens. Chose singulière, il ne semble pas que ce soit dans les temps les plus reculés qu'on se soit fait de ce mal l'idée, sinon la plus fausse, du moins la plus funeste à ceux que le mal atteignait. Si l'on voyait en eux des victimes de la colère divine, on les regardait aussi souvent comme des êtres chers à la Divinité; de toutes façons on les respectait: ils étaient atteints d'une maladie sacrée. Cette manière de penser et d'agir se rencontre encore dans certaines contrées de l'Orient, où la personne d'un fou est, quoi qu'il fasse, inviolable aux yeux de tous.

Les mœurs et les idées du moyen âge, en donnant certaines formes particulières aux manies des fous, en fournissant de nouveaux objets à leur délire, modifièrent les préjugés de la foule au détriment de fous eux-mêmes. L'opinion publique s'égara davantage sur leur compte, et la nouvelle erreur eut les plus fatales conséquences. Comme on croyait à la magie et à la sorcellerie, aux démons et aux possessions, on regarda naturellement comme des magiciens, des sorciers ou des possédés, ceux-là mêmes qui, dans leur délire, croyaient assister au sabbat, se disaient et se croyaient possédés du démon. Au lieu de voir dans les fous des esprits égarés ou des malades, au lieu de chercher, inutilement sans doute, à dissiper leurs illusions par le raisonnement, à les persuader de leur erreur, ce furent ces esprits égarés, ces fous eux-mêmes qui persuadèrent les hommes sensés, leur firent partager l'erreur où ils étaient sur leur propre état. On les crut en réalité ce qu'ils disaient être, et, le plus consciencieusement du monde, après les avoir exorcisés, on les brûla par toute I'Europe. Au commencement du dix-septième siècle, Pierre Delancre (Dieu ait pitié de son âme, car il ne savait ce qu'il faisait), conseiller au parlement de Bordeaux, qui fit en cette qualité bien des autos-da-fé, énumère dans de volumineux ouvrages toute le ruses et malignité de démons et des sorciers (Delancre, 1622). Le procès de Loudun est contemporain du Discours de la méthode. Sous le grand roi, au milieu de tant de lumières éclairant à la fois les lettres, les arts et les sciences, le préjugé qui faisait volontiers de tous les fous autant de sorciers ou de possédés, fut battu en brèche par Malebranche, 


\section{CLÁSSICOS DA PSICOPATOLOGIA}

La Bruyère, Fénelon. L'opinion publique changea encore une fois, mais sans s'éclairer beaucoup davantage, et surtout sans beaucoup améliore le sort des fous. Regardés désormais comme de malades incurables et frappés d'un fléau incompréhensible, traités comme des animaux immondes ou féroces, chargés de chaines dans d'étroits et infects cabanons, rendus furieux par ces traitements, ils auraient à peine eu lieu de regretter, s'ils avaient eu conscience de leur état, les préjugés du moyen âge. Enfin Pinel vint, et le premier en Europe fit tomber les chaînes des fous enfermés à Bicêtre et à la Salpêtrière, et les traita comme des personnes humaines; il se fit l'avocat de ces malheureux et plaida devant la société leur réhabilitation dans les rangs de l'humanités souffrante, sinon de l'humanité raisonnable. L'impulsion donnée par Pinel et par Esquirol se propage; les cabanons deviennent des asiles, les petites maisons des palais, les furieux se calment, les fous se guérissent, bon nombre de jeunes médecins se vouent à l'étude spéciale de ce mal inconnu, une branche importante de la médecine se détache et pousse vigoureusement sous le nom de médecine mentale.

La révolution opérée par Pinel dans les choses, dans le traitement de la folie, a eu sans doute un grand retentissement dans le public; mais les idées répandues aujourd'hui dans le monde sur la folie en sont-elles devenues beaucoup plus nettes et beaucoup plus justes? Il ne le semble pas. Les anciens préjugés sont renversés définitivement dans l'esprit du plus grand nombre, mais ils n'ont pas encore été remplacés généralement par des croyances saines et réfléchies; en disparaissant, ils n'ont guère laissé que le vide. On a applaudi à l'œuvre de Pinel; mais, comme si l'on était satisfait de voir les pauvres fous entourés de soins dévoués et intelligents, on ne se donne pas la peine de s'éclairer sur leur état. Pour la plupart, un asile d'aliénés, une maison de fous est une sorte de pandémonium, où il ne se dit, ne se pense ou ne se fait rien de raisonnable, il ne reste plus rien dans l'aliéné du vieil homme, de l'homme sensé; les fous sont rayés de la liste de hommes; un miracle, ou peu s'en faut, est seul capable de les rendre à la raison et à la société.

Un visiteur mondain parcourt-il les différents quartiers d'un asile, est-il témoin des manies si variées où s'égare la raison humaine, il porte témérairement les jugements les plus erronés sur l'origine, la cause de ces délires, de leurs formes et de leurs objets. Un fou se croit riche, grand seigneur, prince, roi, Dieu lui-même; c'est quelque ambitieux sans doute à qui un amour effréné des richesses et de la puissance a fait perdre l'esprit, dont l'imagination délirante prend pour des réalités dont il jouit les grandeurs échappées à ses désirs. Il n'en est rien: c'est un petit huissier de province qui n'avait 
pas même l'ambition d'être notaire de son village; il se croit roi sur un trône, il n'est qu'un pauvre fou paralysé. Une jeune fille tient-elle des discours obscène, manifeste-t-elle quelque brutale passion; c'est sans doute une femme perdue qui porte le peines de sa débauche, ou tout au moins quelque amante délaissée par un infidèle. Vous vous trompez encore; vous avez devant vous une chaste nonne, une innocente enfant, dont la vie est sans tache, le cœur sans passion, le corps sans souillure; et cependant

\section{C'est Venus tout entière à sa proie attachée}

mais une Vénus hideuse et plus impitoyable que celle qui poursuivait Phèdre, et qui fait sans haine et sans vengeance bien plus de victimes.

Homme ou femme, jeune ou vieille, une personne semble depuis quelque temps changer de caractère, ses sentiments sont autres, ses goûts differents, ses idées légèrement exaltées; elle est triste, elle est rêveuse. Sa famille qui ne s'explique pas ce changement, s'étonne et s'inquiète; nous, qui ne sommes que ce qu'on appelle dans le monde des connaissances ou même des amis, nous remarquons à peine cette altération insensible et n'en cherchons pas la cause. Un jour cette personne disparait; nous nous informons, on nous répond qu'elle à la campagne ou qu'elle voyage. L'air contraint et embarrassé de ceux qui nous parlent, la façon dont ils cherchent à détourner notre pensée sur un autre objet, nous apprennent qu'il y a quelque chose dont on veut faire mystère. Cette campagne, c'est une maison de santé; ce voyage, un innocent mensonge; l'absent est un malade dont la folie s'est enfin révélée manifestement par un violent accès. Mais pourquoi ces détours, ces mensonges, ces précautions pour cacher à tous le mal et sa nature? On dirait que la folie est une maladie honteuse, que les victimes en sont des coupables, qu'il faille blâmer plutôt que plaindre.

Bon nombre de gens redoutent de passer, surtout le soir, auprès d'un cimetière, de voir à découvert le visage d'un mort ou de toucher son linceul; je ne sais quelle terreur qu'ils savent déraisonnable s'empare de leur esprit; leur semble que les morts vont se lever et se précipiter à leur poursuite; et, s'ils n'ont pu éviter le spectacle redouté, mille fantômes, mille angoisses les assaillent longtemps pendant leur sommeil. Cette impression que la mort ou le mort lui-même fait sur quelques esprits, le spectacle de la folie la produit plus forte encore en des âmes qui ne s'émeuvent pas toujours facilement. Je comprends tous ces sentiments, $\mathrm{j}$ 'excuse toutes ces terreurs, je conçois toutes ces fausses idées; mais ne devrait-on pas, au lieu de donner aux victimes de la folie une pitié stérile, au lieu de détourner au plus tôt se regards et sa pensée du triste spectacle qu'elle nous offrent, chercher à s'éclairer un peu sur la 


\section{CLÁSSICOS DA PSICOPATOLOGIA}

nature d'un mal qui semble si effrayant, familiariserons esprit avec cette chose mystérieuse, agiter quelques-uns des problèmes si intéressants et si importants qu'elle soulève? Le terrible n'est que l'inconnu; dès que le jour commence à paraitre, s'évanouissent les fantômes et les terreurs de la nuit.

L'ignorance du public en matière de folie est extrême et incompréhensible; c'est un fait qu'il faut constater et dont nous rechercherons les causes, dussions-nous faire le procès à tout le monde, au public, aux médecins et aux philosophes. Elle se confond et trouve en partie sa raison dans l'ignorance générale où vit le plus grand nombre de tout ce qui touche à la médecine. Le voyageurs nous rapportent que les peuples orientaux regardent volontiers comme des médecins tous les habitants de l'Occident. Cependant de tous les arts celui de guérir, de toute les sciences la science médicale est nécessairement la moins infuse. Ce privilège que l'Orient nous accorde à tous quelque habile praticien de l'Occident. Cette généralisation naïve et spontanée qui fait un indistinctement tient sans doute à l'incapacité ou au charlatanisme des empiriques de ces nations barbares ou déchues, et à la bonne fortune qui de temps à autre a conduit dans ces contrées médecin d'un touriste français, d'un membre de l'école d'Athènes, savant en grec, et requis pour accoucher une femme, outre qu'elle est fort peu logique, nous confère une science qui, en France particulièrement est loin d'être aussi vulgaire. Il n'est point de science, au contraire, qui soit demeurée plus secrète, qui soit moins tombée dans le domaine public, que la médecine et tout ce qui s'y rapporte.

11 peut sembler déraisonnable d'en accuser notre indifférence, car avant même la richesse nous plaçons la santé. Et cependant, si incompréhensible qu'elle puise être, notre indifférence est pour beaucoup dans notre ignorance. Peu de gens cherchent à se connaitre eux-mêmes, à savoir ce qu'est leur esprit, s'il est immortel. Il n'y en a pas beaucoup plus qui cherchent à connaitre leur corps. On mange sans se soucier de savoir comment on digère, on a l'estomac malade et l'on se croit mal au cœur, on éternue et on croit bonnement avoir un rhume au cerveau. Les nobles du moyen âge ne se donnaient point la peine d'apprendre à lire ou à écrire; ils avaient des chapelains ou des secrétaires pour leur faire la lecture et rédiger leurs rares messages; lire et écrire, c'était l'affaire de ces gens-là. Nous aussi, nous avons des savants pour s'occuper de notre machine et savoir ce qui s'y passe, les médecins; chacun son métier. En bonne santé, qu'a-t-on besoin de savoir comment on digère? On n'en digérerait pas mieux. Malade, on a recours aux habiles.

L'incrédulité n'est pour rien dans l'ignorance du grand nombre. L'incrédulité est bonne dans une comédie, mais non dans la vie réelle. 
L'incrédulité à la médecine est à peu près comme l'incrédulité à Dieu, superficielle. Quand on se porte bien et quand on est heureux, on peut blasphémer Dieu et se rire des médecins tout à son aise; mais quand la douleur nous accable et que la mort approche, il faut un sceptique ou un athée bien endurci pour ne pas appeler le médecin et Dieu à son secours. D'ailleurs, à côté de l'art de guérir qu'on peut juger plus ou moins puissant, et qui est particulièrement une profession, il y a dans la médecine la science du corps humain, qui généralement n'est contestée par personne et peut être possédée par beaucoup.

Faut-il s'en prendre à la volonté jalouse des médecins eux-mêmes? Non pas précisément, mais on accuserait à bon droit leurs habitudes, leur langage et leurs écrits. Il est juste de dire que les médecins eux-mêmes écartent le public profane, autant que celui-ci s'éloigne spontanément de leur science. Voyez un-médecin au lit de son malade; comme les paroles tombent rares de sa bouche! Voyez ses consultations et ses ordonnances; ce sont de véritables oracles pour la brièveté du langage et l'obscurité des signes. Sa tenue, sa démarche, son silence, sa parole, tout réprime notre curiosité, et nous fait presque regarder la médecine comme une science secrète et mystérieuse. Il y a certainement plusieurs raisons excellentes de ces mœurs médicales; la prudence, l'intérêt du malade lui-même commande souvent cette réserve et ce ton d'autorité. Il y en a aussi de moins bonnes et de purement historiques. La médecine se ressent encore aujourd'hui de son antique origine; nous sommes loin du temps où l'art de guérir était exercé par des prêtres, où les hôpitaux étaient des temples, mais nous sommes encore voisins de celui où Guénaut en robe et en bonnet pointu éclaboussait Boileau en passant sur sa mule, de celui où le médecin était reconnaissable à sa perruque à marteaux. Il serait déraisonnable de vouloir que la médecine devint une science généralement répandue, mais il y a dans les sciences médicales des connaissances élémentaires qui sont l'a b c de la médecine, et que la plupart ne savent même pas épeler. Certaines parties de la médecine pourraient être vulgarisées dans le monde, et la médecine, à son tour, pourrait sortir un peu de cet aparté où elle se complaît.

Il résulte, en effet, de cette situation isolée de la science médicale plusieurs conséquences fâcheuses pour le public et pour les médecins eux-mêmes. Personne, à part les médecins, ne lit les ouvrages des médecins; ce sont comme des livres sacrés écrits par des adeptes et pour les adeptes de l'art de guérir. A en juger par le nombre des volumes qui se publient chaque année, aucune science ne devrait faire plus de progrès que la médecine. Mais nulle part on ne semble avoir moins de soucis des conditions qui font un bon 


\section{CLÁSSICOS DA PSICOPATOLOGIA}

livre. Ce que les médecins donnent et prennent trop souvent pour un livre n'est qu'une série d'observations cliniques longuement développées, suivies quelquefois de chiffres pour toute doctrine. Voilà encore une des causes qui nous éloignent de la lecture des ouvrages médicaux; il est rare qu'un livre soit vraiment bon quand il n'est que savant. Il y a dans les sciences médicales, comme partout, des procédés de composition et d'exposition, sinon de style, qui donnent à la doctrine, sinon plus de vérité, au moins plus de lumière. Ce qualités sont singulièrement négligées par nos médecins; ils les acquerraient bien vite s'ils écrivaient quelquefois pour nous, et nous irions à eux plus volontiers pour nous instruire, s'ils voulaient faire quelques frais pour nous avoir comme lecteurs.

A qui que ce soit qu'il en faille attribuer la cause, le monde est trop ignorant de ce qui touche la science médicale. Au moins y a-t-il certains sujets d'un intérêt plus vif, certains maux comme la folie, qui jetant à la fois le désordre dans le corps et dans l'esprit du malade, soulevant des questions de droit et de morale, devraient exciter tout particulièrement la curiosité des gens du monde, des légistes, de philosophes, des moralistes. Il n'en est rien cependant, et cette fois les médecins sont hors de cause.

Un homme du monde, parce qu'il est à peu près dans son bon sens, parce qu'il se sent maître de sa raison et de sa volonté, ne conçoit guère qu'il en puisse jamais perdre l'empire. Il lui semble qu'il saura toujours, lui, retenir sa raison, si quelque catastrophe morale ou physique venait à la menacer; oubliant que cette raison qu'il croit si bien tenir lui échappe chaque fois qu'il se livre au sommeil, oubliant avec quelle facilité le buveur la laisse au fond de son verre. Il se croit sujet tout comme un autre à la fièvre, mais non pas à la folie. Le magistrat, fort de sa connaissance des lois qui protègent la liberté des individus contre les captateurs d'héritage, et la famille contre les erreurs e et les fureurs de l'aliéné, s'imagine trop souvent que rien ne lui sera plus facile que d'appliquer en toutes circonstances les termes de la loi, sans erreurs et sans injustice, qu'il pourra toujours distinguer dans son interrogatoire un homme raisonnable d'un insensé, que toujours il pourra décider en sûreté de conscience si l'homicide est un crime ou l'effet d'un délire, si le testateur jouissait assez de son bon sens pour disposer librement et raisonnablement de sa fortune. Il se repose pour cela sur sa raison à lui, sur son propre bon sens: il ne croit pas nécessaire de faire de la folie un étude plus savante.

Il est juste de décharger les médecins de toute responsabilité á ce sujet; tout en reconnaissant combien est légitime l'intervention du magistrat dans les affaires de l'aliéné, ils ne cessent de réclamer contre la légèreté de beaucoup 
de décisions judiciaires, de signaler dans un bon nombre de questions l'incompétence de juges qui, si intelligents qu'ils soient, n'ont que leur bon sens pour guide. Ils demandent chez les magistrats de connaissances plus profondes, les médecins une part plus grande dans la solution des procès que la folie embarrasse. Quelques-uns vont jusqu'à proposer qu'un cours d'aliénation mentale sois annexé aux cours des écoles de droit, pour familiariser les magistrats avec un mal en présence duquel ils doivent se trouver fréquemment dans leur carrière, de même que la médecine légale est enseignée spécialement aux futurs médecins. Ceux-là oublient que ce cours d'aliénation mentale qu'ils réclament pours l'instruction des étudiants en droit n'existe pas même encore pour les étudiants en médecine. D'ailleurs l'institution d'un pareil enseignement serait difficile, et le remède certainement inefficace. Ne serait-il pas plus simple et plus utile de vulgariser certaines questions que la folie fait naitre? Cette fois c'est affaire aux philosophes au moins autant qu'aux médecins, qui ont surtout pour objet la guérison son du mal lui-même.

Si les livres des médecins n'ont guère de lecteurs hors du monde médical, les écrits des philosophes en ont un peu davantage, des ouvrages philosophiques traitant des facultés mentales de l'aliéné, discutant les plus intéressantes questions qu'un tel sujet soulève en dehors de la physiologie pure, de la pathologie, de la thérapeutique corporelle, en trouveraient peut-être beaucoup. Mais des tels ouvrages n'existent pas, c'est donc aux philosophes qu'il faut s'en prendre, ils sont seuls et grandement coupables. Quand bien même la guérison de l'aliéné appartiendrait au seul médecin, connaitre ou étudier l'état de son esprit appartient au philosophe, c'est son droit et ce serait son devoir. L'étude des égarements de la folie éclairerait sans aucun doute le philosophe sur la conduite de la saine raison; la connaissance des lois de l'esprit, de la marche des passions, de l'imagination, de la volonté, des fonctions des sens, chez l'homme en possession de son bon sens, éclairerait aussi l'étude du délire de la folie. Si le philosophe pouvait savoir ce que le fou conserve de raison, ce qu'il possède encore de liberté et de moralité, à quelle condition un homme cesse d'être responsable devant la justice humaine ou divine des actes dont il est l'auteur; s'il faisait part de ses découvertes, ou seulement de ses pensées, de ses doutes au médecin qui soigne le malade, au juge qui interroge le meurtrier, à l'avocat qui le défend, au juré qui décide de sa vie ou de sa mort, au magistrat qui a le pouvoir d'interdire l'aliéné de ses droits, de le priver de sa liberté corporelle, de donner force de loi à l'expression des dernières volontés d'un testateur ou de les casser comme inspirées par le délire; s'il disait aux gens du monde qu'un fou et toujours un homme; 


\section{CLÁSSICOS DA PSICOPATOLOGIA}

s'il appliquait, s'il réussissait à faire comprendre comment on peut perdre ainsi la raison, comment le phénomènes de folie se rattachent à ceux de la raison droite, ne serait-il pas une œuvre utile et que l'on est en droit d'attendre de la philosophie moderne? Mais ce livre n'existe pas, et il n'existera pas encore quand nous aurons achevé ces recherches, car il faudrait plus que la bonne volonté, plus que le travail, plus que le vif intérêt que ces études nous inspirent, pour mener à bonne fin une tâche si difficile.

Les philosophes eux-mêmes par leur silence semblent avoir mis les fous au ban de l'humanité. Il en est un cependant qui, sur l'invitation d'un professeur éminet de la Faculté de Médecine de Paris, entreprit une étude philosophique de l'aliénation mentale, pour éclairer des lumières de la psychologie les leçons physiologiques de M. A. Royer-Collard. Mais les Nouvelles Considérations sur les rapports du physique et du moral de Maine de Biran, bien qu'elles renferment beaucoup de chose instructives et une certaine somme de vérités, sont un livre de métaphysique, où l'observation directe de la folie est remplacée le plus souvent par le raisonnement, et que domine l'esprit d'un système exclusif, étroit et préconçu. Personne plus que Maine de Biran n'a mis l'aliéné hors des rangs de la société, hors de la portée des observations du psychologue, et renvoyé l'étude de la folie à la seule physiologie. Comme on définit souvent l'homme un être raisonnable et libre, et que l'insensé, l'aliéné est défini à son tour par ces noms mêmes, un être qui n'a pas sa raison, qui ne jouit pas de sa liberté; comme Maine de Biran, renchérissant sur ces définitions, faisait consister l'action et la pensée dans ce qu'il appelait le compos e le conscium sui, un fou, pour lui, n'était plus un homme, mais un être sans pensée véritable, presque sans âme, un monstre une créature déclassée, en tout cas embarrassante.

De ce silence ou de cette manière de considérer la folie, il résulte que la philosophie qui devait étudier l'état mental de l'aliéné, ne fût-ce qu'à titre de ........ épreuve des lois de la logique et de la morale, parait au contraire avoir repoussé cette étude comme étrangère ou indifférente; que la psychologie qui devait aider la physiologie à plaider devant le monde en faveur de l'aliéné et à connaitre la nature de son mal, semble s'être déchargée de ce devoir et l'avoir laissé tout entier à la médecine. Cette abstention ne doit pas durer. Si les philosophes, oubliant que Descartes était un des physiologistes les plus savants de son temps, se sont tenus trop loin des physiologistes, si les physiologistes de leur côté, tout entiers aux recherches expérimentales qui leur ont fait faire tant de belles découvertes, se sont aussi trop éloignés de philosophes, si, pendant une partie de ce demi-siècle, il y a eu séparation 
entre les deux sciences et presque guerre comme entre deux partis, il est temps que l'étude de l'aliénation mentale réunisse en commun les efforts des médecins et des philosophes. Puisque le mal quelle qu'en soit la nature, qu'on appelle folie, jette le désordre dans les phénomènes de la sensibilité, de l'intelligence, de la volonté, dans le jeu de toutes ces facultés, est un mot, qui sont du ressort de la psychologie, il faut que les psychologues étudient la sensation, la passion, la raison, la volonté troublées par la folie, comme ils les étudient saine et régulières. Ce n'est pas trop des efforts réunis des médecins et des philosophes pour répandre sur un tel sujet une lumière suffisante. A l'honneur des médecins, il est juste de dire que ce sont eux qui ont fait les premiers pas et demandé le concours de la philosophie. Considérant l'aliénation mentale comme un objet digne d'une étude toute spéciale, qu'il importe de séparer du reste de la médecine, pour qu'il ne se confonde pas, obscur et négligé, dans le cercle immense de tous les maux qui nous affligent, ils ont fondé à Paris, depuis près de vingt ans, une sorte d'académie privée dont l'étude de l'aliénation mentale est l'unique objet. Comprenant que, pour bien expliquer le dérèglement des facultés de l'esprit, il est nécessaire d'en bien connaître le jeu régulier, ils ont invité à s'unir à eux des psychologues, et donné à leur académie le nom significatif de Société médico-psychologique. Dans les séances de cette société se discutent les plus intéressantes questions sur la folie et les états analogues de l'esprit ou du corps; et la philosophie y dit son mot. L'union est donc établie, il ne s'agit plus que de la rendre étroite, complète et durable. C'est travailler à cette réconciliation nécessaire de la philosophie et de la physiologie, en même temps que c'est toucher un des sujets le plus intéressants et les moins explorés des philosophes, que d'instituer quelques recherches philosophiques sur la folie.

Nous ne prétendons ni traiter, ni surtout résoudre tous les problèmes que soulève l'alienation mentale dans l'esprit du psychologue, du moraliste ou du légiste; nous voudrions seulement poser en termes clairs et précis les questions les plus importantes, et exprimer sur chacune d'elles considérations communes ou personnelles dont on appréciera la valeur.

Les principales questions que la philosophie peut aider la médecine à résoudre, et qui sont comme le complément nécessaire de la psychologie et de la morale, nous paraissent être les suivantes et naître les unes des autres dans l'ordre suivant.

Une première question se propose naturellement à l'esprit du physiologiste ou du psychologue: La folie est-elle une maladie corporelle, ou est-elle rigoureusement, selon l'expression consacrée prise à la lettre, une maladie 


\section{CLÁSSICOS DA PSICOPATOLOGIA}

mentale? Est-ce un mal qui frappe immédiatement le corps ou qui afflige directement l'esprit? Une maladie de quelque organe, du cerveau par exemple, ou une maladie de l'âme? Un fou est-il un malade comme un autre, comme un homme atteint d'un fièvre cérébrale; ou bien n'est-ce qu'une intelligence qui se trompe, comme celui qu'on appelle aussi un insensé, parce qu'il commet une erreur grossière de jugement ou de raisonnement? La folie a-t-elle son siégé, son principe et son foyer exclusif dans le corps ou dans l'esprit?

On comprend, à l'énoncer seulement, toute l'importance de cette première question, et l'on aperçoit d'avance les conséquences philosophiques et médicales qui résulteront de la solution qu'elle recevra. Si le fou n'est qu'un esprit qui se trompe, le philosophe devra reconnaitre que l'esprit et sujet, comme le corps, à certaines maladies qui lui sont propres et peuvent l'envahir spontanément; et le médecin devra abandonner la cure de cette intelligence malade, puisqu'il n'est que le guérisseur de la machine corporelle, aux soins du logicien qui connait mieux les lois de la pensée, la nature des sophisme et le moyen de les corriger. Il faudra laisser de côté les médicaments de toute sorte, et guérir par des procédés psychologiques un mal qui s'attaque à l'âme elle-même.

Il n'est pas au-dessus des forces de la physiologie et de la psychologie réunies de prouver que l'affaiblissement ou l'égarement des facultés mentales est toujours l'effet immédiat d'un mal qui affecte l'économie animale, quelqu'une de ses partie ou de ses fonctions; que la folie n'est jamais une maladie propre de l'esprit qui naîtrait spontanément dans l'âme des seuls incidents de la vie intellectuelle ou morale; que le fou est toujours un corps malade dont la raison ne s'égare que par une conséquence de l'union générale et étroite de l'âme et du corps, et des corrélations particulières et mystérieuses qu'a établies la nature entre certains organes corporels et les facultés mentales.

Toutes les folies ne se ressemblent pas; rien, au contraire, n'est plus variable que la forme et l'objet du délire chez les différents malades. Chez l'un, toutes les facultés mentales sont affaiblies, chez l'autre, elles sont surexcitées; chez celui-ci le désordre ou l'affaiblissement ne s'observe que dans l'exercice de certaines facultés particulières, chez celui-là le délire ne porte que sur un ordre d'idées fort restreint. L'un est le jouet d'hallucinations de toutes sorte, tous ses sens en sont assignés à la fois ou successivement; son caractère habituel, se sentiments naturels sont changés complétement, il ne sait plus juger de rien avec rectitude; ses discours incessants ressemblent à la lecture à haute voix des mots épars d'un vocabulaire; point d'apparence de raisonnement, de liaison quelconque entre les idées, ou bien des déductions 
que la logique ne pourrait classer dans aucune catégorie de sophismes; ses souvenirs sont anéantis ou confondus, ses actions n'ont plus ni motif, ni but apparent; le délire a envahi son esprit tout entier. L'autre, au contraire, juge sainement de la plupart des choses, il n'extravague que sur la cause des douleurs qu'il ressent. Celui-ci a perdu la mémoire, celui-là la conserve fidèle et entière, si ce n'est qu'il ne peut se rappeler aucun nom propre, pas même le sien; le délire de l'un inoffensif, l'autre est poussé, malgré lui, par une force inconnue à l'homicide. Toutes les facultés mentales de l'aliéné paraissent donc simultanément ou séparément troublées par la maladie, sans ordre et sans règle; il semble que dans la folie règne un hasard aveugle qui trouble ou respecte capricieusement toutes les fonctions intellectuelles.

Le philosophe ne doit-il pas rechercher avec le physiologiste si ce désordre même n'est pas gouverné par quelques lois secrètes? Il devra tenter $\mathrm{d}$ 'expliquer comment une modification morbide de l'économie ou de quelque organe peut produire ces hallucinations qui assiègent l'esprit du fou, abolir ou troubler une faculté particulière et respecter les autres, pousser invinciblement la meilleure des mères à donner la mort à ses enfants. Il cherchera si quelque puissance de l'esprit n'est pas plus facile à emporter ou ne résiste pas plus longtemps à la folie envahissante, si l'on ne peut pas saisir quelque ordre régulier suivant lequel les facultés mentales succombent le plus souvent l'une après l'autre à la maligne influence. Il faut, en un mot, que le philosophe étudie l'état de la sensibilité, de l'intelligence et de la volonté chez l'aliéné.

Si la raison ne s'égare que parce que le trouble de certaines fonctions organiques a, en vertu des lois de l'union du corps et de l'esprit, son retentissement dans l'âme, et jette le désordre dans les facultés intellectuelles, est-ce à dire que l'altération des organes, cause directe de la folie, ait elle-même toujours et infailliblement une cause physique, par exemple, une blessure à la tête, ou un vice de constitution du cerveau, ou une maladie accidentelle de cet organe, ou une maladie antérieure de quelque autre partie du corps, en un mot, un concours ou un enchainement de circonstances et d'accidents purement matériels? Ou bien cette altération organique qui porte le trouble dans le facultés de l'esprit n'est-elle pas souvent produite, à son tour, par de cause de l'ordre moral? Une forte émotion, un profond chagrin, une passion violente ne peut-elle, en vertu de l'influence réciproque de l'âme sur les organes, jeter le désordre dans l'économie vitale, ébranler assez puissamment le cerveau pour produire dans le corps un tel état, qu'il produise à son tour, par contrecoup, le dérèglement des facultés mentales? Une mère, par exemple, voit son enfant périr violemment sous ses yeux, un père de famille est précipité tout à coup, 


\section{CLÁSSICOS DA PSICOPATOLOGIA}

avec les siens, par un hasard ou par une faute personnelle, de l'opulence dans l'extrême misère: il ne se peut pas que son esprit, même sous l'empire d'une violente émotion, délire spontanément et s'égare à jamais; mais ne se peut-il pas que, frappé de terreur ou agité de remords, il imprime au système cérébral et nerveux une secousse qui lui enlève subitement la raison, ou que la douleur produise lentement une altération des fonctions et de organes corporels qui cause elle-même insensiblement l'affaissement ou le trouble des facultés de l'esprit? Cette nouvelle question n'intéresse pas moins manifestement le philosophe que le médecin, et la solution en doit être demandée également à la physiologie et à la psychologie. La manière dont les morales agissent concurremment avec les causes physiques pour produire la folie a des conséquences trop considérables et trop évidentes, pour que l'importance en puisse échapper à personne.

La diversité que l'on observe dans les affections corporelles et les formes du délire chez les aliénés avait déjà suggéré aux anciens la pensée d'établir des distinctions dans la folie et d'en classer les différentes espèces. Pinel proposa à son tour une classification plus complète, modifiée, augmentée surtout par ses successeurs, mais dont les principaux traits furent généralement conservés. N'y-a-t-il pas lieu de se demander quels principes généraux doivent présider à une classification de ce genre: si, par exemple, le désordre des facultés intellectuelles avant pour cause immédiate le trouble de l'économie animale, ce n'est pas dans la seule différence des affections organiques qu'il faudrait chercher le principe de toute division, sans tenir compte ni de l'objet, ni de la nature du délire, ni des facultés de l'esprit dont la folie s'est emparée, ou bien s'il ne conviendrait pas de prendre pour base de cette classification l'objet même du délire et les facultés égarées, enfin si ces deux éléments, et peut-être quelque autre encore, ne doivent pas intervenir et dans quelles proportions? Quoi qu'il en puisse être, la philosophie a certainement, comme la physiologie, quelques questions à résoudre ou à méditer, quelques considérations à proposer sur ce sujet d'une grande importance pour le traitement de la folie et les augures que l'on peut tirer de son heureuse ou malheureuse terminaison.

Ce n'est point en effet pour séparer les aliénés du reste des hommes et mettre la société à l'abri des dangers dont leur folie la menace, qu'on les confine ainsi dans les asiles mais pour traiter et, s'il est possible, pour guérir leur mal. Si le traitement qu'on pratique, qu'on a pratiqué de tout temps, que l'on peut pratiquer, ne consistait que dans l'usage exclusif des médicaments pharmaceutiques ou des moyens qui agissent directement sur le corps, 
le psychologue n'aurait rien à dire, ni presque rien à penser du traitement de la folie: il n'aurait qu'à constater que la thérapeutique physique ou pharmaceutique peut seule agir sur une maladie toute corporelle. Mais de tout temps, les médecins, guidés par le bon sens, ont pensé que l'on devait agir aussi sur l'esprit de l'aliéné; qu'on devait s'adresser, selon les cas particuliers, à sa sensibilité souvent excessive, au débris de raison ou de volonté qui lui reste, à son imagination, à la puissance secrète mais positive que les phénomène moraux exercent sur les dispositions des organes, pour calmer le délire, en changer le cours, combattre l'erreur, ramener peu à peu l'ordre et la santé dans l'économie corporelle, et partant réintégrer l'esprit dans la possession de son bon sens et de sa liberté. Quelque fois même l'usage de cette médecine intellectuelle ou morale a été préconisé avec excès et pratiqué sans mesure, comme étant seul capable de guérir la folie. Le psychologue qui doit avoir étudié l'influence qu'exerce, même sans l'intervention de la volonté, l'esprit sur les organes, aussi bien que celle que l'état des organes exerce réciproquement sur l'esprit, qui a dû reconnaitre que dans certains cas la folie peut être produite ou tout au moins occasionnée par une cause morale jetant d'abord le trouble dans les organes et par contre-coup dans les facultés mentales, pourrait éclairer le médecin sur les moyens de ce genre qui avéraient le plus de chances de succès, sur le cas généraux où il conviendrait de les employer, sur la manière dont ils peuvent agir. En effet, si le médecin récusait absolument sur cette matière la compétence du psychologue ou du moraliste, il récuserait la moitié de sa propre science. C'est par ce qu'il est connait lui-même de l'empire de passions, des sentiments de toute espèce sur l'état de organes, du mode d'action et des limites de ce pourvoir, en un mot, c'est parce que il est lui-même psychologue ou moraliste en même temps que médecin, que tantôt il essaye de faire servir cette influence à la guérison de malades, et tantôt la néglige comme évidemment impuissante. Le traitement moral ou intellectuel de la folie, sa puissance, la manière d'en faire usage, le discernement de cas généraux où il convient le mieux de l'appliquer, ce que l'on peut en attendre, voilà un nouveau sujet de recherches et de méditations pour le philosophe.

Il est encore un ordre de question tout différent mais aussi important, qui appelle l'examen du moraliste, et dont les recherches précédentes devront éclairer l'étude. La foi française a prévu dans sa sagesse les cas principaux où peut se trouver un membre de la société, particulièrement les cas d'aliénation mentale, et dans sa justice elle a rendu certaines décisions qui protègent l'aliéné ou le privent de quelques droits qu'il possédait avant l'invasion de son mal, qu'il recouvrera s'il vient à recouvrer la raison. Il appartient donc 


\section{CLÁSSICOS DA PSICOPATOLOGIA}

au moraliste d'examiner quelle est la situation de l'aliéné devant la loi, si la loi est suffisamment protectrice des droits de toute espèce qui appartiennent à l'individu, de ceux qu'elle conserve à l'aliéné, de ceux de sa famille et de la société tout entière, si la loi, supposée sage et équitable en principe, l'est également dans tous les cas qui peuvent se présenter, si l'application en est facile, si les instruments n'en faussent pas bien souvent l'esprit et l'intention, s'il ne serait pas possible d'éviter un certain nombre des erreurs et des anomalies que l'expérience de tous le jours révèle et que la raison déplore. Par exemple, qui est chargé, qui est capable d'appliquer la loi? Le magistrat. Qui est capable et chargé de connaître l'état de malade? Le médecin. Voilà deux autorités, deux juges, dont les idées ne sont pas les mêmes, dont la science est autre et la compétence différente. Un conflit en peut résulter, chaque jour en fournit des preuves. Une famille demande l'interdiction d'un de ses membres, parce qu'il est aliéné. Qui refuse ou prononce l'interdiction? Un magistrat, cela est juste. Qui déclare l'individu fou ou sain d'esprit? Le médecin? Non, c'est encore le magistrat; le médecin n'est que consulté mais c'est l'homme de loi qui décide la question de folie comme la question d'interdiction, et peut ne pas suivre l'avis du médecin. Est-ce également juste? Un homme a tué son semblable; on le soupçonne de folie, ou la défense le fait passer pour fou; qui l'absout ou le condamne? Un jury de citoyens; rien de plus juste. Mais qui juge de l'état de son esprit, de sa folie, ou de son bon sens? C'est encore le jury qui consulte le médecin, mais décide selon son inspiration personnelle. Est-ce également juste? Ne peut-on rien faire pour protéger plus efficacement soit les aliénés contre les dangers d'une erreur de la justice, soit la société contre ses propres erreurs et les conséquences du délire de fous?

Telle sont les principales questions qui doivent appeler l'attention du philosophe et du moraliste, du légiste et du magistrat.

\section{References}

Delancre, P. Tableau de l'inconscience de mauvais anges et démons, etc., in $-4^{\circ}$. Paris, 1613.

Delancre, P. L'Incrédulité et mécréance du sortilège pleinement convaincue, etc., in- $4^{\circ}$. Paris, 1622. 
Citação/Citation: Lemoine, A. (2018, março). L'aliené devant da Philosophie, la morale et la societé. Revista Latinoamericana de Psicopatologia Fundamental, 21(1), 124-144. http:// dx.doi.org/10.1590/1415-4714.2018v21n1p124.9

Editores do artigo/Editors: Prof. Dr. German E. Berrios

Recebido/Received: 20.12.2017/ 12.20.2017 Aceito/Accepted: 15.1.2018 / 1.15.2018

Copyright: (C) 2009 Associação Universitária de Pesquisa em Psicopatologia Fundamental/ University Association for Research in Fundamental Psychopathology. Este é um artigo de livre acesso, que permite uso irrestrito, distribuição e reprodução em qualquer meio, desde que o autor e a fonte sejam citados / This is an open-access article, which permits unrestricted use, distribution, and reproduction in any medium, provided the original authors and sources are credited.

\section{Albert Lemoine (1824-1874)}

Professor de filosofia no Licée Bonaparte.

This is an open-access article, which permits unrestricted use, distribution, and reproduction in any medium for non-commercial purposes provided the original authors and sources are credited. 